\title{
Finite-Time Integral Sliding Mode Control for Motion Control of Permanent-Magnet Linear Motors
}

\author{
Xiaohui Mo ${ }^{1,2}$ and Qixun Lan ${ }^{2,3}$ \\ ${ }^{1}$ Department of Information Technology, Jinling Institute of Technology, Nanjing 211169, China \\ ${ }^{2}$ School of Automation, Southeast University, Nanjing 210096, China \\ ${ }^{3}$ School of Mathematics and Physics, Henan University of Urban Construction, Pingdingshan 467000, China
}

Correspondence should be addressed to Qixun Lan; qixun407@163.com

Received 26 March 2013; Accepted 29 May 2013

Academic Editor: Lijun Zhang

Copyright (C) 2013 X. Mo and Q. Lan. This is an open access article distributed under the Creative Commons Attribution License, which permits unrestricted use, distribution, and reproduction in any medium, provided the original work is properly cited.

\begin{abstract}
The finite-time motion control problem of permanent-magnet linear motor (PMLM) is studied in this paper. Firstly, based on finite-time integral sliding mode (FTISM) technique, a finite-time control (FTC) law is proposed such that the PMLM can track the desired trajectory in finite time in the presence of disturbances. Secondly, to alleviate the chattering caused by discontinuous property of the control law, a novel saturation function is introduced to replace the signum function in the proposed FTC law. Finally, the effectiveness of the proposed method is shown by simulation results and comparisons.
\end{abstract}

\section{Introduction}

Permanent-magnet linear motor (PMLM) not only has the merits such as high force density achievable, low thermal losses, high precision and accuracy, and simple mechanical structure [1], but also is probably the most natural choice for applications involving high-speed, high-precision motion control among the electric motor drives [2]. Nowadays, PMLM has been widely used in the precision manufacturing industries. Compared with the traditional rotary motors [38], linear motors require no indirect coupling mechanisms as in gear boxes, chains, and screws coupling, which greatly reduces the contact type nonlinearities and mechanical disturbances such as friction force and backlash $[1,2,9]$. Therefore, PMLMs meet the increasing demands for high performance servo applications and have been successfully used in machine tools [10], semiconductor manufacturing systems [11], and so forth. Since PMLMs are not equipped with transmission mechanisms, the achievable performance of PMLMs, such as the ability to reduce the effects of uncertainties and external disturbances, is unavoidably lost to some extent. Thus, it is very important to reduce these effects, either through proper mechanical design or via control schemes. The effects may be kept to admissible levels, through the alternative mechanical design approach, but they may be very costly one. It may be an economical feasible method to suppress these effects based on the controller design for PMLM system.

The friction (Coulomb, viscous, and stiction) and ripple forces (detent and reluctance forces) are the two major disturbances that affect the system directly [1]. Friction is a natural phenomenon that arises from the contact between the translator and the track [12]. Moreover, in a closedloop control system, the friction may result in a steadystate error, a limit cycle, and a low bandwidth. Ripple forces are strong, position-dependent forces arising from the magnetic structure of a PMLM. It comprises of the cogging force and reluctance force, both of which are periodic relationship with respect to the position of the translator relative to the magnets [2]. With the growing interest in improving the precision of position/speed tracking control, more advanced disturbance rejection method is needed in the PMLM control system. To improve the disturbance rejection performance of PMLMs, numerous methods have 
been presented in the recent literature. Based on sliding mode control and a proportional-integral-based disturbance observer, [13] proposes a control law to guarantee the stability of the tubular linear synchronous motor system regardless of the payload mass. In [11], an Elman neural network controller based on improved particle swarm optimization is developed for a PMLM. In [1], a robust control law which consisted of feedback control law based on integral sliding mode (ISM) method and feedforward compensation part based on adaptive estimation algorithm is proposed. In [2], a new hysteretic relay method is proposed to identify the force ripples and friction in PMLM system, based on which a composite control approach which is consisted of a PID control law with a feedforward compensation term is proposed. Reference [9] proposed a state-periodic adaptive compensation approach, which uses one trajectory period past information along the state axis to update the current adaptation law for PMLM system. In [14], to achieve high control performance, a field-programmable gate array based intelligent complementary sliding mode control is proposed for the PMLM system such that the mover can track periodic reference trajectories. To solve the precision control problem of PMLM motion control system in the presence of significant disturbance, a robust control scheme based on the disturbance observer (DOB) is proposed to reduce the sensitivity of the control performance to disturbance [15]. To provide adequate disturbance rejection performance and overcome the stability problem as well as improve the motion control accuracy, [16] proposes a control approach which combines feedback feedforward controller and a digital DOB for a PMLM drive system. All the above mentioned control methods $[1,2,9,13-16]$ can improve disturbance rejection performance of PMLMs in some extent. However, most of the existing results $[1,2,9,14]$ about PMLMs motion control laws are asymptotically stable ones. This implies that the convergence rate of the system states is at best exponential. In other words, the desired position and velocity cannot be tracked in a finite time. Obviously, the control laws with finite-time convergence are more desirable. Besides faster convergence rates, the closed-loop systems under finite-time control (FTC) laws usually demonstrate higher accuracy and better disturbance rejection properties [17-21]. Due to the above superiorities, FTC technique has been used in robotic manipulator systems $[18,20]$, inverted pendulum systems [21], spacecraft systems [22], PMSM systems [23], and multiagent systems $[24,25]$, and so forth.

In this paper, the motion control problem of PMLM is considered in the presence of friction and ripple forces. By introducing a finite-time integral sliding mode (FTISM) manifolds, a FTISM control law is proposed for PMLM system, which can guarantee that the desired trajectories can be tracked in finite time. However, the proposed FTISM control law has a discontinuous term which leads to chattering. Inspired by traditional boundary layer method, a novel saturation function is introduced to replace the signum function in the proposed discontinuous control law. Analysis indicates that under the proposed continuous FTISM control law, the trajectories of the system will converge to a much smaller region than that of under the traditional boundary method; that is, compared with the traditional boundary layer approach, the closed-loop system under the proposed continuous FTISM control law has much smaller steady-state errors. The major merit of the proposed method is that it can not only effectively alleviate chattering, but also maintain relatively good disturbance rejection performance. All the results are supported by simulations.

\section{Problem Statement and Preliminaries}

The mathematical model of a PMLM can be approximately described as follows [9]:

$$
\ddot{x}(t)=-\frac{k_{f} k_{e}}{R m} \dot{x}(t)-\frac{1}{m} F_{\text {fric }}-\frac{1}{m} F_{\text {ripple }}+\frac{k_{f}}{R m} u(t),
$$

where $u(t)$ is time-varying motor terminal voltage, $x(t)$ is the motor position, $R$ is the resistance, $m$ is the moving thrust block mass, $k_{f}$ is the force constant, $k_{e}$ is the back electromotive force, and $F_{\text {fric }}$ and $F_{\text {ripple }}$ denote the friction and ripple force, respectively.

The friction force affecting the movement of the translator may be modeled as a combination of Coulomb friction, viscous friction, and the component due to Stribeck effect, which can be interpreted as stiction. The friction force is modeled as

$$
F_{\text {fric }}=\left[f_{c}+\left(f_{s}-f_{c}\right) e^{-\left(\dot{x} / \dot{x}_{s}\right)^{2}}+f_{v} \dot{x}\right] \operatorname{sign}(\dot{x}),
$$

where $f_{c}$ is the Coulomb friction coefficient, $f_{s}$ is static friction coefficient, $f_{v}$ is the viscous friction coefficient, and $\dot{x}_{s}$ is the lubricant parameter. The ripple force can be described by a sinusoidal function of the load position. In this paper, we assume that the ripple force could be any kind of Fourier expansion such as

$$
F_{\text {ripple }}=\sum_{i=1}^{\infty} A_{i} \sin \left(\omega_{i} x+\varphi_{i}\right)
$$

where $A_{i}$ is the amplitude, $\omega_{i}$ is the state-dependent ripple force frequency, and $\varphi_{i}$ is the phase angle [9].

Let $a=-k_{f} k_{e} /(R m), b=k_{f} /(R m)$, and $d(t)=F_{\text {fric }}+$ $F_{\text {ripple }}$ denote lumped disturbance, and let $x_{1}(t)=x(t)$ and $\dot{x}(t)=x_{2}(t)$ denote position and velocity, respectively, then we have

$$
\begin{gathered}
\dot{x}_{1}(t)=x_{2}(t) \\
\dot{x}_{2}(t)=a x_{2}(t)+b u(t)-\frac{d(t)}{m} .
\end{gathered}
$$

Define the tracking errors

$$
\begin{aligned}
& e_{1}(t)=x_{1}(t)-x_{d}(t), \\
& e_{2}(t)=x_{2}(t)-\dot{x}_{d}(t),
\end{aligned}
$$

where $x_{d}(t)$ and $\dot{x}_{d}(t)$ are the desired position and velocity, respectively. The control objective is to track the given desired position $x_{d}(t)$ and the corresponding desired 
velocity $\dot{x}_{d}(t)$ in finite time, that is, to design an FTC law for the following error system:

$$
\begin{gathered}
\dot{e}_{1}(t)=e_{2}(t) \\
\dot{e}_{2}(t)=a e_{2}(t)+b u(t)-\frac{d(t)}{m}+a \dot{x}_{d}(t)-\ddot{x}_{d}(t)
\end{gathered}
$$

such that $e_{1}(t) \rightarrow 0$ and $e_{2}(t) \rightarrow 0$ in finite time.

Next, we review the concept of finite-time stability and several lemmas, which will serve as basis for this study.

Definition 1 (see [19]). Consider system

$$
\dot{x}=f(x), \quad f(0)=0, \quad x \in R^{n},
$$

where $f(\cdot) \quad: \quad D \rightarrow R^{n}$ is nonLipschitz continuous on a neighborhood $D$ of the origin $x=0$ in $R^{n}$. The equilibrium $x=0$ of (7) is (locally) finite-time stable if there are an open neighborhood $U$ of the origin and a function $T: U\{0\} \rightarrow[0, \infty)$, such that every solution trajectory $x\left(t, x_{0}\right)$ of system (7) starting from the initial point $x_{0} \in U\{0\}$ is well defined and unique in forward time for $t \in\left[0, T\left(x_{0}\right)\right)$ and $\lim _{t \rightarrow T\left(x_{0}\right)} x\left(t, x_{0}\right)=0$. Here $T\left(x_{0}\right)$ is called the convergence time (of the initial state $x_{0}$ ). The equilibrium of system (7) is finite-time stable if it is Lyapunov stable and finite-time convergent. If $U=D=R^{n}$, the origin is a globally finite-time stable equilibrium.

Lemma 2 (see [17]). Assume that there exists a continuous function $V(x): U \rightarrow R$ such that the following conditions hold.

(i) $V(x)$ is positive definite.

(ii) There exist real numbers $c>0$ and $\alpha \in(0,1)$ and an open neighborhood $U_{0} \subset U$ of the origin such that $\dot{V}(x)+c V^{\alpha}(x) \leq 0, x \in U_{0} \backslash\{0\}$.

Then the origin is a finite-time stable equilibrium of system (7), and the finite convergence time $T$ satisfies $T \leq$ $V(x(0))^{1-\alpha} / c(1-\alpha)$. If $U=U_{0}=R^{n}$, the origin is a globally finite-time stable equilibrium of (7).

Lemma 3 (see [26]). The origin of system

$$
\dot{x}=y, \quad \dot{y}=u
$$

is a globally finite-time-stable equilibrium under the feedback control law

$$
u=-k_{1} \operatorname{sig}^{\alpha_{1}}(x)-k_{2} \operatorname{sig}^{\alpha_{2}}(y)
$$

where $k_{1}, k_{2}>0,0<\alpha_{1}<1$ and $\alpha_{2}=2 \alpha_{1} /\left(1+\alpha_{1}\right)$.

\section{Main Results}

Assumption 4. Assume that both the friction force $F_{\text {fric }}$ and the ripple force $F_{\text {ripple }}$ are bounded, and there exists a positive constant scalar $l$ such that $\left|F_{\text {fric }}+F_{\text {ripple }}\right| \leq l$, that is, $|d(t)| \leq$ l.
For system (6), a finite-time integral sliding mode is designed as [21]

$$
s=e_{2}+\int_{0}^{t}\left(k_{1} \operatorname{sign}\left(e_{1}\right)\left|e_{1}\right|^{\alpha_{1}}+k_{2} \operatorname{sign}\left(e_{2}\right)\left|e_{2}\right|^{\alpha_{2}}\right) d \tau,
$$

where $k_{1}, k_{2}>0, \alpha_{1}>0$, and $\alpha_{2}=2 \alpha_{1} /\left(1+\alpha_{1}\right)$.

Theorem 5. If system (6) satisfies Assumption 4, then under the control law

$$
\begin{gathered}
u(t)=-\frac{1}{b}\left(k_{1} \operatorname{sign}\left(e_{1}\right)\left|e_{1}\right|^{\alpha_{1}}+k_{2} \operatorname{sign}\left(e_{2}\right)\left|e_{2}\right|^{\alpha_{2}}\right. \\
\left.+a e_{2}+a \dot{x}_{d}-\ddot{x}_{d}+\eta \operatorname{sign}(s)\right)
\end{gathered}
$$

with $s$ defined in (10), and $\eta>l / m$, and the states of system (6) will be stabilized to the origin in finite time; that is, $e_{1} \rightarrow$ $0, e_{2} \rightarrow 0$ in finite time.

Proof. Consider the Lyapunov function

$$
V=\frac{1}{2} s^{2}
$$

By differentiating $V$ with respect to time $t$, we have

$$
\begin{aligned}
\dot{V}= & s \dot{s} \\
= & s\left(a e_{2}+b u-\frac{d(t)}{m}+a \dot{x}_{d}-\ddot{x}_{d}\right. \\
& \left.\quad+k_{1} \operatorname{sign}\left(e_{1}\right)\left|e_{1}\right|^{\alpha_{1}}+k_{2} \operatorname{sign}\left(e_{2}\right)\left|e_{2}\right|^{\alpha_{2}}\right) .
\end{aligned}
$$

Substituting (11) into (13) yields

$$
\begin{aligned}
\dot{V} & =s \dot{s}=\left(-\frac{d(t)}{m}-\eta \operatorname{sign}(s)\right) s \leq-\left(\eta-\frac{l}{m}\right)|s| \\
& =-\sqrt{2}(\eta-l) V^{1 / 2} .
\end{aligned}
$$

By Lemma 2, (14) implies that the FTISM $s=0$ can be reached in finite time.

As $s=0$, we have

$$
e_{2}=-\int_{0}^{t}\left(k_{1} \operatorname{sign}\left(e_{1}\right)\left|e_{1}\right|^{\alpha_{1}}+k_{2} \operatorname{sign}\left(e_{2}\right)\left|e_{2}\right|^{\alpha_{2}}\right) d \tau,
$$

that is,

$$
\ddot{e}_{1}=-k_{1} \operatorname{sign}\left(e_{1}\right)\left|e_{1}\right|^{\alpha_{1}}-k_{2} \operatorname{sign}\left(e_{2}\right)\left|e_{2}\right|^{\alpha_{2}} .
$$

According to Lemma 3, the states of system (6) will converge to zero in finite time. This completes the proof.

Remark 6. The proposed control law (11) can not only suppress the lumped disturbance (friction and ripple forces) effectively, but also guarantee that the states of closed-loop error system is stabilized to origin in finite time. However, the signum function in control law (11) leads to undesired 
chattering of the closed-loop system. In order to eliminate chattering, the signum function

$$
\operatorname{sign}(s)= \begin{cases}\frac{s}{|s|}, & |s| \neq 0, \\ 0, & |s|=0\end{cases}
$$

can be replaced by the saturation function

$$
\operatorname{sat}\left(\frac{s}{\epsilon}\right)= \begin{cases}\frac{s}{|s|}, & |s| \geq \epsilon, \\ \frac{s}{\epsilon}, & |s|<\epsilon,\end{cases}
$$

where $\epsilon>0$ represents the width of the boundary layer [20], according to the conventional method. In this manner, we obtain a continuous control law that can be expressed as

$$
\begin{gathered}
u(t)=-\frac{1}{b}\left(k_{1} \operatorname{sign}\left(e_{1}\right)\left|e_{1}\right|^{\alpha_{1}}+k_{2} \operatorname{sign}\left(e_{2}\right)\left|e_{2}\right|^{\alpha_{2}}\right. \\
\left.+a e_{2}+a \dot{x}_{d}-\ddot{x}_{d}+\eta \operatorname{sat}\left(\frac{s}{\epsilon}\right)\right),
\end{gathered}
$$

which can guarantee the finite time reachability to the region $|s| \leq \Delta_{1} \triangleq l \epsilon /(m \eta)<\epsilon($ as $\eta>l / m)$.

Next, we introduce a class of novel nonlinear saturation functions as follows:

$$
\operatorname{sat}_{\alpha}(x)= \begin{cases}\operatorname{sign}(x), & |x|>1 \\ \operatorname{sign}(x)|x|^{\alpha}, & |x| \leq 1\end{cases}
$$

where $0 \leq \alpha \leq 1$. It is easy to show that

$$
\operatorname{sat}_{\alpha}(x)= \begin{cases}\operatorname{sign}(x), & \alpha=0 \\ \operatorname{sat}(x), & \alpha=1 .\end{cases}
$$

In the case of $x \in\{x|0<| x \mid<1\}$ and $0<$ $\alpha<1$, we have $\mid$ sat $_{\alpha}(x)|>| \operatorname{sat}(x) \mid$, and sat ${ }_{\alpha}(x)=$ sat $(x)$ as $|x|=1$ or $x=0$. In order to facilitate comprehension, the standard saturation function sat $(x)$, the classical signum function sign $(x)$, and the novel saturation function sat ${ }_{\alpha}(x)$ with two different $\alpha(1 / 3$ and $2 / 3)$ are shown in Figure 1. It can be observed from Figure 1 that $\mid$ sat $_{\alpha}(x)|>|$ sat $(x) \mid$ as $x \in\{x|0<| x \mid<1\}$ and sat $_{\alpha}(x)=\operatorname{sat}(x)$ as $|x| \geq 1$ or $x=0$, which verifies the validity of the above analysis.

Theorem 7. If system (6) satisfies Assumption 4, then under the control law

$$
\begin{gathered}
u(t)=-\frac{1}{b}\left(k_{1} \operatorname{sign}\left(e_{1}\right)\left|e_{1}\right|^{\alpha_{1}}+k_{2} \operatorname{sign}\left(e_{2}\right)\left|e_{2}\right|^{\alpha_{2}}\right. \\
\left.+a e_{2}+a \dot{x}_{d}-\ddot{x}_{d}+\eta \operatorname{sat}_{\alpha}\left(\frac{s}{\epsilon}\right)\right),
\end{gathered}
$$

with $s$ defined in (10), $\eta>l / m$, and

$$
\operatorname{sat}_{\alpha}\left(\frac{s}{\epsilon}\right)= \begin{cases}\operatorname{sign}\left(\frac{s}{\epsilon}\right), & |s| \geq \epsilon, \\ \operatorname{sign}\left(\frac{s}{\epsilon}\right)\left|\frac{s}{\epsilon}\right|^{\alpha}, & |s|<\epsilon\end{cases}
$$

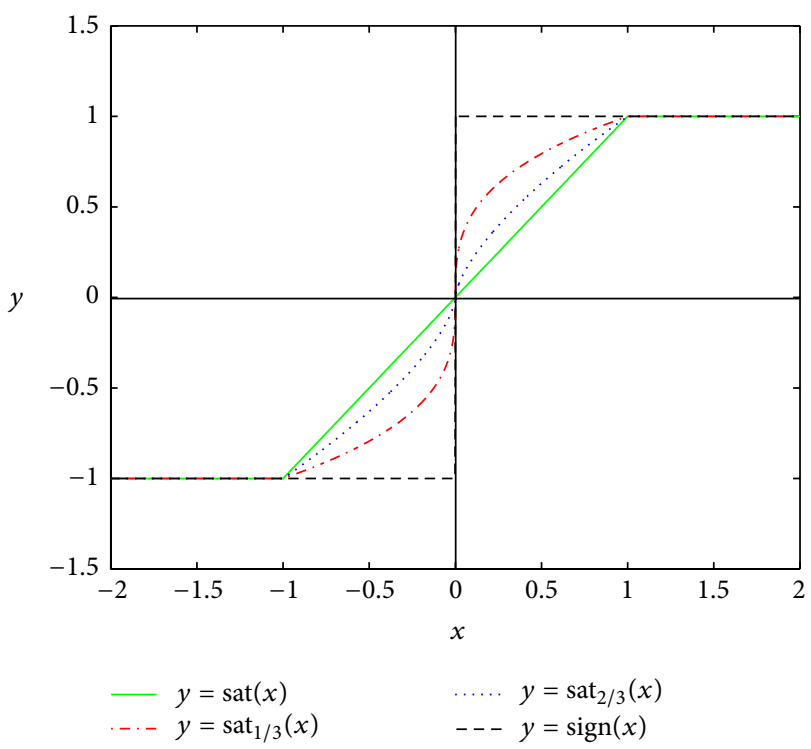

FIGURE 1: Classical signum functions sign $(x)$, standard saturation functions sat $(x)$ and sat $\alpha(x)$ with different $\alpha$.

with $0<\alpha<1$ and $\epsilon>0$, and the trajectory of system (6) will converge to the neighborhood of FTISM $s=0$ as

$$
|s| \leq \Delta_{2}, \quad \Delta_{2}=\left(\frac{l}{m \eta}\right)^{1 / \alpha} \epsilon
$$

in finite time.

Proof. Substituting control law (22) into (13) yields

$$
\begin{aligned}
& \dot{V}=s \dot{s}=\left(d(t)-\eta \operatorname{sat}_{\alpha}\left(\frac{s}{\epsilon}\right)\right) s \\
& =\left\{\begin{array}{l}
\left(d(t)-\eta \operatorname{sign}\left(\frac{s}{\epsilon}\right)\right) s \\
\left(d(t)-\eta \operatorname{sign}\left(\frac{s}{\epsilon}\right)\left|\frac{s}{\epsilon}\right|^{\alpha}\right) s,
\end{array}\right. \\
& \leq\left\{\begin{array}{l}
-\left(\eta-\frac{l}{m}\right)|s| \\
-\left(\eta \frac{|s|^{\alpha}}{\epsilon^{\alpha}}-\frac{l}{m}\right)|s|
\end{array}\right. \\
& = \begin{cases}-\sqrt{2}\left(\eta-\frac{l}{m}\right) V^{1 / 2}, & |s| \geq \epsilon \\
-\sqrt{2}\left(\eta \frac{|s|^{\alpha}}{\epsilon^{\alpha}}-\frac{l}{m}\right) V^{1 / 2}, & |s|<\epsilon .\end{cases}
\end{aligned}
$$

By Lemma 2, we can have that the region $\Delta_{2}=$ $(l / m \eta)^{1 / \alpha} \epsilon$ can be reached in finite time. This completes the proof.

Remark 8. One may argue that through adjusting control gains $\eta$ to be large enough, both convergence regions, that is, $\Delta_{1}$ and $\Delta_{2}$ can be rendered to be as small as desired, which means that the proposed novel saturation 


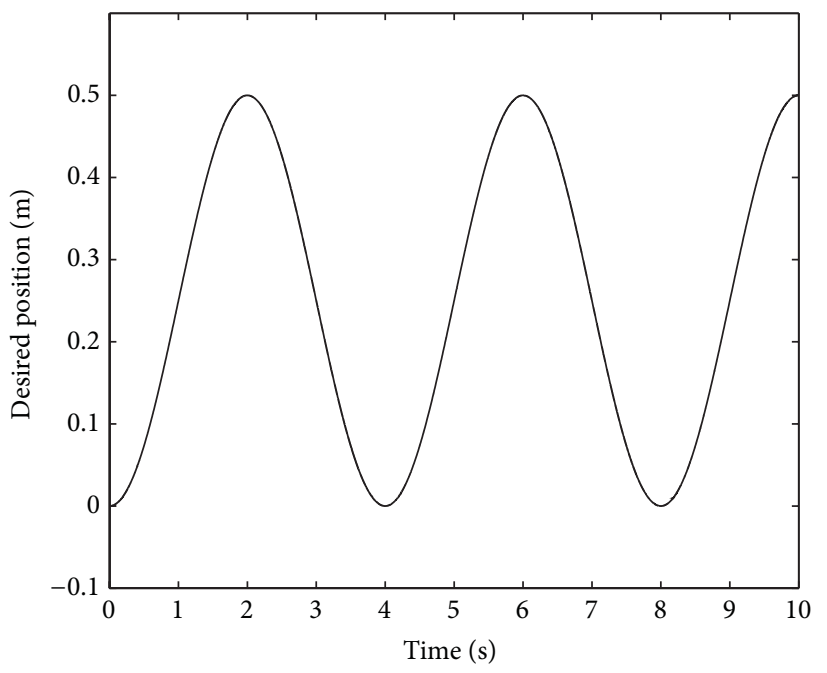

(a)

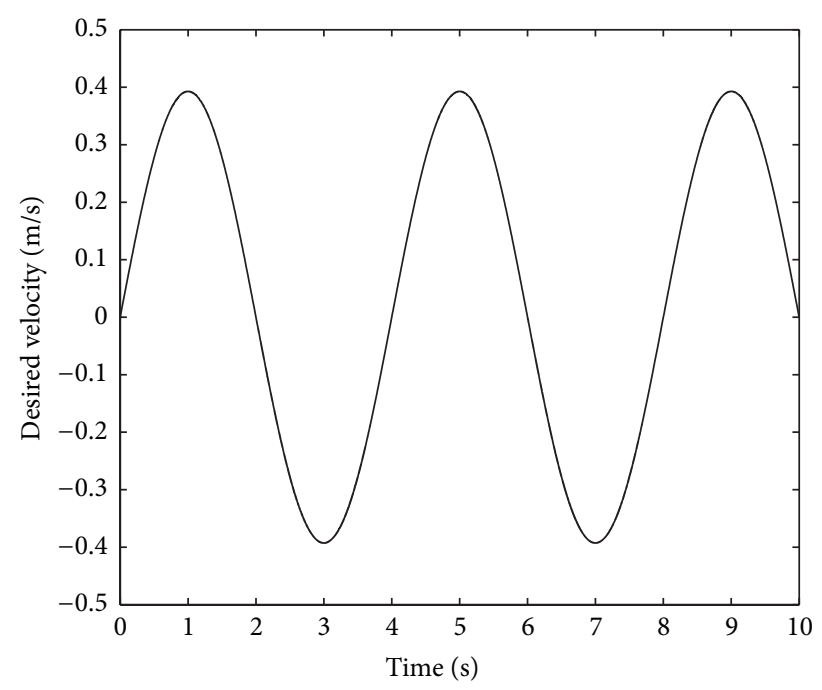

(b)

Figure 2: The desired position (a) and velocity (b).

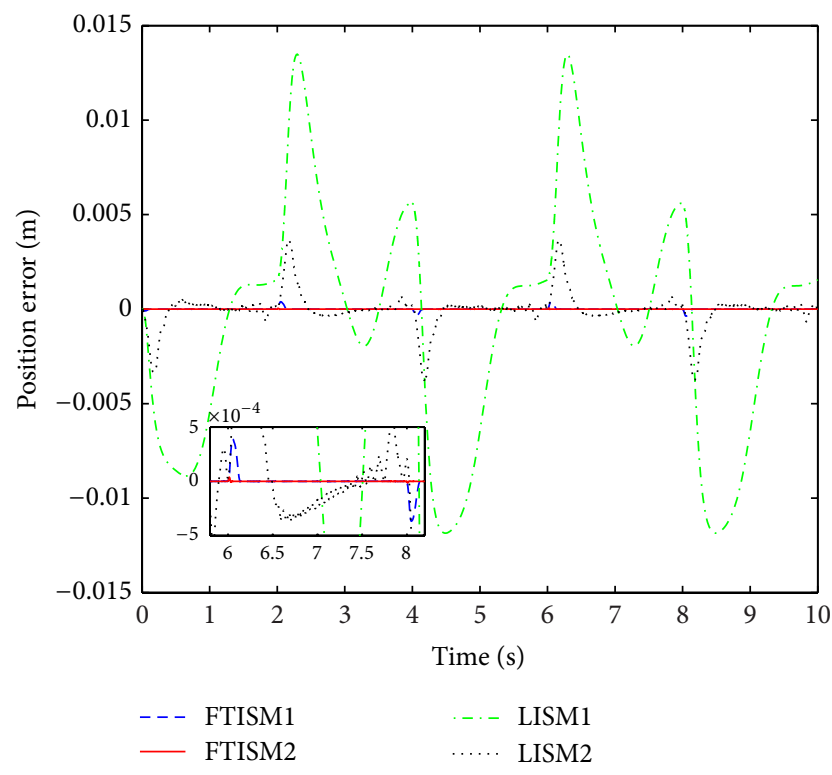

Figure 3: Position tracking errors under control laws (19), (22), (27), and (28).

function sat ${ }_{\alpha}(\cdot)$ does not have any prominent advantage over the standard saturation function sat $(\cdot)$ disturbance rejection performance. However, high-gain feedback control system often exhibits instability in the actual operation. From the considerations as well as control saturation constraints, $\eta$ cannot be selected to be sufficiently large. In this case for the control law (22) has an additional parameter $\alpha$ that can be adjusted to enhance the disturbance rejection performance without obvious increase in the control input. In fact, $0<l /(m \eta)<1$, we can select $\alpha$ to approximate to 0 such that $1 / \alpha$ in (24) is sufficiently larger than 1 , which means $(l /(m \eta))^{1 / \alpha} \epsilon \ll(l /(m \eta)) \epsilon$. That is, $\Delta_{2}$ can be designed to be much smaller than $\Delta_{1}$, which implies that we can regulate the parameters $\alpha$ and $\eta$ such that under the control law (22), the closed-loop system can have smaller steady-state errors compared to that under the control law (19).

Remark 9. Obviously, if $\alpha_{1}=1$ in the proposed FTISM (10), then it degenerates to the traditional linear integral sliding mode (LISM), and the FTISM control law (11) becomes

$$
u(t)=-\frac{1}{b}\left(k_{1} e_{1}+k_{2} e_{2}+a e_{2}+a \dot{x}_{d}-\ddot{x}_{d}+\eta \operatorname{sign}(s)\right) .
$$

It is easy to prove that under the control law (26), the states of system (6) will be stabilized to the origin asymptotically. Similarly, in order to alleviate chattering of the LISM control law (26), we take saturation functions (18) and (23) to replace the signum function in the LISM control law (26), respectively. We obtain two continuous LISM control law:

$$
\begin{aligned}
& u(t)=-\frac{1}{b}\left(k_{1} e_{1}+k_{2} e_{2}+a e_{2}+a \dot{x}_{d}-\ddot{x}_{d}+\eta \text { sat }\left(\frac{s}{\epsilon}\right)\right), \\
& u(t)=-\frac{1}{b}\left(k_{1} e_{1}+k_{2} e_{2}+a e_{2}+a \dot{x}_{d}-\ddot{x}_{d}+\eta \operatorname{sat}_{\alpha}\left(\frac{s}{\epsilon}\right)\right)
\end{aligned}
$$

for system (6). And under continuous control laws (27) and (28), the states of the system (6) will converge to the small regions $\Delta_{1}$ and $\Delta_{2}$, respectively, in finite time.

\section{Simulations}

In this section, we take a PMLM as target to illustrate the effectiveness of the proposed methods. The model parameters of PMLM are given as $m=5.4 \mathrm{~kg}, R=16.8 \mathrm{ohms}, k_{f}=$ 
TABLE 1: Control parameters for the simulation.

\begin{tabular}{lc}
\hline Control laws & Parameters \\
\hline FTISM1 & $k_{1}=25, k_{2}=10, \alpha_{1}=1 / 3, \alpha_{2}=1 / 2, \eta=10, \epsilon=0.5$ \\
FTISM2 & $k_{1}=25, k_{2}=10, \alpha_{1}=1 / 3, \alpha=1 / 2, \alpha=1 / 3, \eta=10, \epsilon=0.5$ \\
LISM1 & $k_{1}=25, k_{2}=10, \eta=10, \epsilon=0.5$ \\
LISM2 & $k_{1}=25, k_{2}=10, \eta=10, \alpha=1 / 3, \epsilon=0.5$ \\
\hline
\end{tabular}

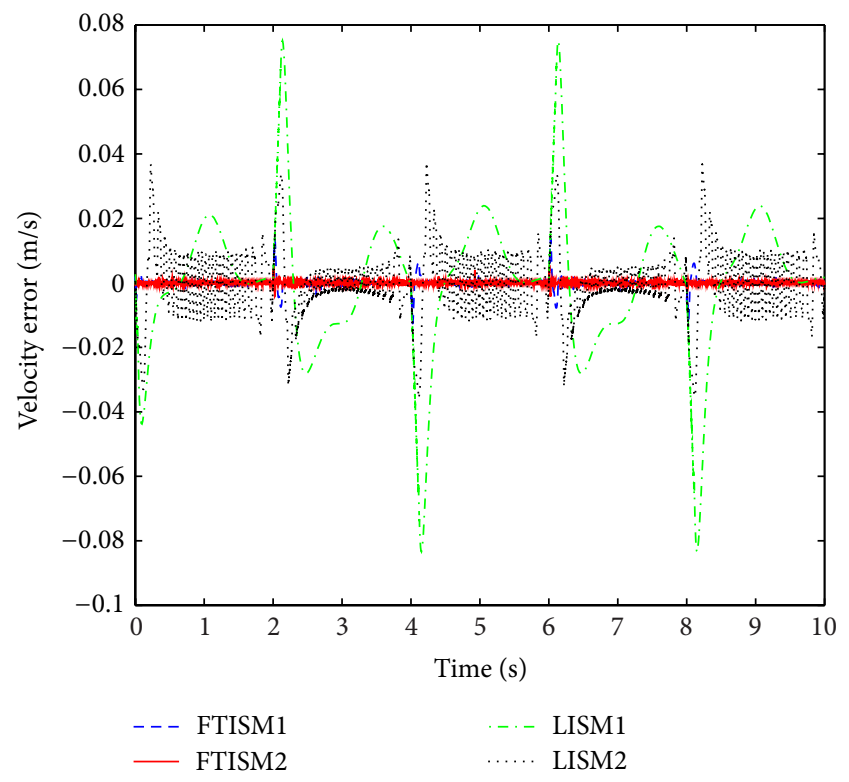

FIGURE 4: Velocity tracking errors under control laws (19), (22), (27), and (28).

$130 \mathrm{~N} / \mathrm{A}$, and $k_{e}=123 \mathrm{~V} / \mathrm{m} / \mathrm{s}$, and the same values are used in [9]. Among the friction forces, the viscous force has the greatest impact to PMLM control system, which is proportional to the speed in the motion process [27], and the parameters for the friction are taken as $f_{c}=10 \mathrm{~N}, f_{s}=$ 20 , and $f_{v}=10 \mathrm{~N}$. To represent the high order Fourier expansion, the ripple force is chosen as

$$
F_{\text {ripple }}=A_{1} \sin (\omega x)+A_{2} \sin (3 \omega x)+A_{3} \sin (5 \omega x),
$$

where $A_{1}=8.5, \omega=314 \mathrm{rad} / \mathrm{m}, A_{2}=4.25$, and $A_{3}=2.0$. The following reference trajectory is used [9]:

$$
x_{d}(t)=0.25 \sin \left(2 \pi \frac{t}{T_{d}}-\frac{\pi}{2}\right)+0.25
$$

with $T_{d}=4 \mathrm{~s}$.

For convenience, in the simulation abbreviations FTISM1, FTISM2, LISM1 and LISM2 denote control laws (19), (22), (27), and (28), respectively.

Consider the initial states of system $(4)$ as $\left(x_{1}(0), x_{2}(0)\right)=$ $(0,0)$. The control parameters of all the four control laws (19), (22), (27), and (28) are listed in Table 1. The desired position and velocity are shown in Figure 2 . The position and velocity tracking error curves under control laws (19), (22), (27), and (28) are shown in Figures 3 and 4, respectively. In this

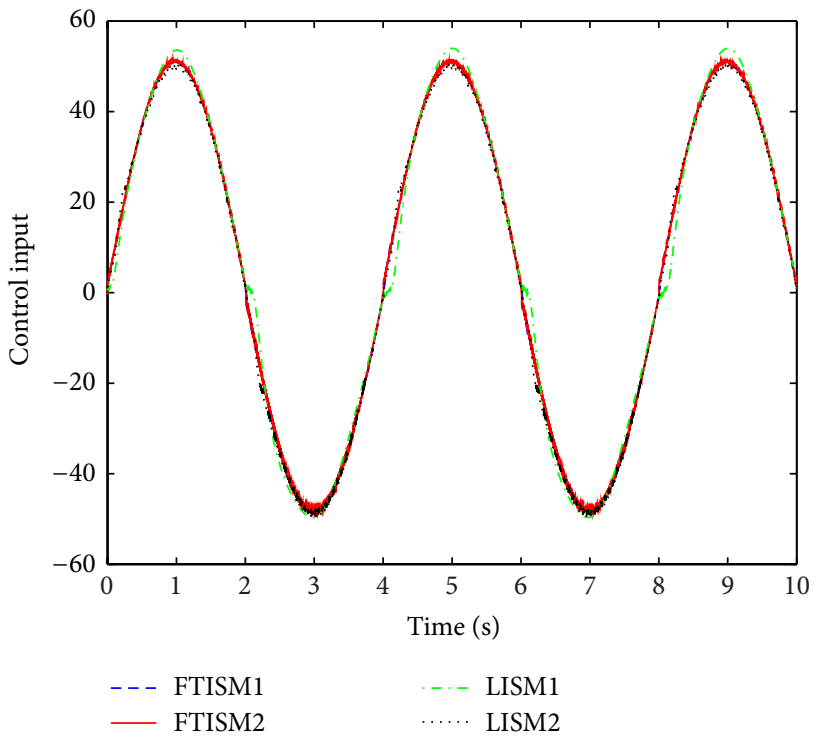

FIGURE 5: Input signals under control laws (19), (22), (27), and (28).

simulation, the switching gain $\eta$ and width of the boundary layer $\epsilon$ for all the four control laws FTISM1, FTISM2, LISM1, and LISM2 are the same. However, as clearly shown in the simulation results, the proposed continuous FTISM control laws FTISM1 and FTISM2 can achieve better control performance than that of the continuous LISM control laws LISM1 and LISM2, and the control laws with novel saturation function FTISM2 and LISM2 can obtain much smaller tracking errors than that with standard saturation function FTISM1 and LISM1. Under the control law FTISM2, the system not only has the best disturbance rejection performance, but also has the fastest convergence rate, which is an evidence as stated in Remark 8. The input signals of control laws FTISM1, FTISM2, LISM1, and LISM2 are shown in Figure 5. It can be observed from Figure 5 that all of the four control laws FTISM1, FTISM2, LISM1, and LISM2 can alleviate chattering effectively, and the amplitudes of the control laws FTISM1, FTISM2 are smaller than that of the control laws LISM1 and LISM2.

\section{Conclusions}

In this paper, a finite-time integral sliding mode (FTISM) control method has been proposed to study the motion control problem of PMLMs. Under the proposed FTISM control law, the desired trajectory can be tracked in finite time in the presence of friction and ripple forces. Note that the signum 
function could lead to chattering. To alleviate chattering, a novel saturation function has been introduced to replace the signum function in the proposed FTISM control law. Both theoretical analysis and simulation results show that under the proposed FTISM control law with the novel saturation function, the trajectories of the system have better control performance (convergence rate and disturbance rejection ability) than that of FTISM control law with the standard saturation function. In summary, the major merit of the proposed method is that it can not only effectively alleviate chattering, but also maintain relatively good disturbance rejection performance.

\section{Acknowledgment}

This work is supported by the Natural Science Foundation of Jiangsu Province, China (Grant no. BK2011089).

\section{References}

[1] K. K. Tan, S. N. Huang, and T. H. Lee, "Robust adaptive numerical compensation for friction and force ripple in permanentmagnet linear motors," IEEE Transactions on Magnetics, vol. 38, no. 1, pp. 221-228, 2002.

[2] S. Chen, K. K. Tan, S. Huang, and C. S. Teo, "Modeling and compensation of ripples and friction in permanent-magnet linear motor using a hysteretic relay," IEEE/ASME Transactions on Mechatronics, vol. 15, no. 4, pp. 586-594, 2010.

[3] S. Li and Z. Liu, "Adaptive speed control for permanent-magnet synchronous motor system with variations of load inertia," IEEE Transactions on Industrial Electronics, vol. 56, no. 8, pp. 30503059, 2009.

[4] S. H. Li and H. Gu, "Fuzzy Adaptive internal model control schemes for PMSM speed-regulation system," IEEE Transactions on Industrial Informatics, vol. 8, no. 4, pp. 767-779, 2012.

[5] H. Liu and S. Li, "Speed control for PMSM servo system using predictive functional control and extended state observer," IEEE Transactions on Industrial Electronics, vol. 59, no. 2, pp. 11711183, 2012.

[6] C. Huang, J. Lin, T. Liao, C. Chen, and J. Yan, "Quasi-sliding mode control of chaos in permanent magnet synchronous motor," Mathematical Problems in Engineering, vol. 2011, Article ID 964240, 10 pages, 2011.

[7] J. Cao, J. Wen, and B. Cao, "Robust ESO two-degree-of-freedom control design for permanent magnet synchronous motor," Mathematical Problems in Engineering, vol. 2010, Article ID 405251, 10 pages, 2010.

[8] J. Yu, J. Gao, Y. Ma, and H. Yu, "Adaptive fuzzy tracking control for a permanent magnet synchronous motor via backstepping approach," Mathematical Problems in Engineering, vol. 2010, Article ID 391846, 13 pages, 2010.

[9] H. Ahn, Y. Chen, and H. Dou, "State-periodic adaptive compensation of cogging and Coulomb friction in permanent-magnet linear motors," IEEE Transactions on Magnetics, vol. 41, no. 1 I, pp. 90-98, 2005.

[10] W. Su and C. Liaw, "Adaptive positioning control for a LPMSM drive based on adapted inverse model and robust disturbance observer," IEEE Transactions on Power Electronics, vol. 21, no. 2, pp. 505-517, 2006.

[11] F.-J. Lin, L.-T. Teng, and H. Chu, "Modified Elman neural network controller with improved particle swarm optimisation for linear synchronous motor drive," IET Electric Power Applications, vol. 2, no. 3, pp. 201-214, 2008.

[12] B. Armstrong-Hélouvry, P. Dupont, and C. C. De Wit, "A survey of models, analysis tools and compensation methods for the control of machines with friction," Automatica, vol. 30, no. 7, pp. 1083-1138, 1994.

[13] F. Cupertino, D. Naso, E. Mininno, and B. Turchiano, "Slidingmode control with double boundary layer for robust compensation of payload mass and friction in linear motors," IEEE Transactions on Industry Applications, vol. 45, no. 5, pp. 16881696, 2009.

[14] F. Lin, J. Hwang, P. Chou, and Y. Hung, "FPGA-based intelligent-complementary sliding-mode control for PMLSM servo-drive system," IEEE Transactions on Power Electronics, vol. 25, no. 10, pp. 2573-2587, 2010.

[15] K. K. Tan, T. H. Lee, H. F. Dou, S. J. Chin, and S. Zhao, "Precision motion control with disturbance observer for pulsewidthmodulated-driven permanent-magnet linear motors," IEEE Transactions on Magnetics, vol. 39, no. 3, pp. 1813-1818, 2003.

[16] M. Yan and Y. Shiu, "Theory and application of a combined feedback-feedforward control and disturbance observer in linear motor drive wire-EDM machines," International Journal of Machine Tools and Manufacture, vol. 48, no. 3-4, pp. 388-401, 2008.

[17] S. P. Bhat and D. S. Bernstein, "Geometric homogeneity with applications to finite-time stability," Mathematics of Control, Signals, and Systems, vol. 17, no. 2, pp. 101-127, 2005.

[18] Y. Feng, X. Yu, and Z. Man, "Non-singular terminal sliding mode control of rigid manipulators," Automatica, vol. 38, no. 12, pp. 2159-2167, 2002.

[19] Y. Hong, J. Wang, and D. Cheng, "Adaptive finite-time control of nonlinear systems with parametric uncertainty," IEEE Transactions on Automatic Control, vol. 51, no. 5, pp. 858-862, 2006.

[20] S. Yu, X. Yu, B. Shirinzadeh, and Z. Man, "Continuous finitetime control for robotic manipulators with terminal sliding mode," Automatica, vol. 41, no. 11, pp. 1957-1964, 2005.

[21] Q. Zong, Z.-S. Zhao, and J. Zhang, "Higher order sliding mode control with self-tuning law based on integral sliding mode," IET Control Theory and Applications, vol. 4, no. 7, pp. 1282-1289, 2010.

[22] H. B. Du and S. H. Li, "Finite-time attitude stabilization for a spacecraft using homogeneous method," Journal of Guidance, and Control, vol. 35, no. 3, pp. 740-748, 2012.

[23] S. H. Li, H. X. Liu, and S. H. Ding, "A speed control for a PMSM using finite-time feedback control and disturbance compensation," Transactions of the Institute of Measurement and Control, vol. 32, no. 2, pp. 170-187, 2010.

[24] S. Li, H. Du, and X. Lin, "Finite-time consensus algorithm for multi-agent systems with double-integrator dynamics," Automatica, vol. 7, no. 8, pp. 1706-1712, 2011.

[25] H. Du, S. Li, and C. Qian, "Finite-time attitude tracking control of spacecraft with application to attitude synchronization," IEEE Transactions on Automatic Control, vol. 56, no. 11, pp. 2711-2717, 2011.

[26] S. P. Bhat and D. S. Bernstein, "Continuous finite-time stabilization of the translational and rotational double integrators," IEEE Transactions on Automatic Control, vol. 43, no. 5, pp. 678-682, 1998.

[27] N. Barabanov and R. Ortega, "Necessary and sufficient conditions for passivity of the LuGre friction model," IEEE Transactions on Automatic Control, vol. 45, no. 4, pp. 830-832, 2000. 


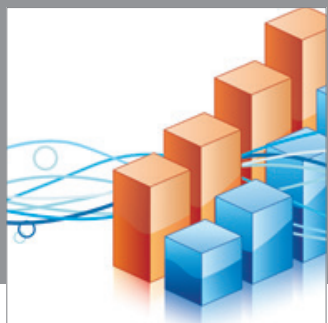

Advances in

Operations Research

mansans

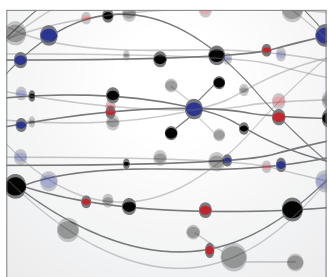

The Scientific World Journal
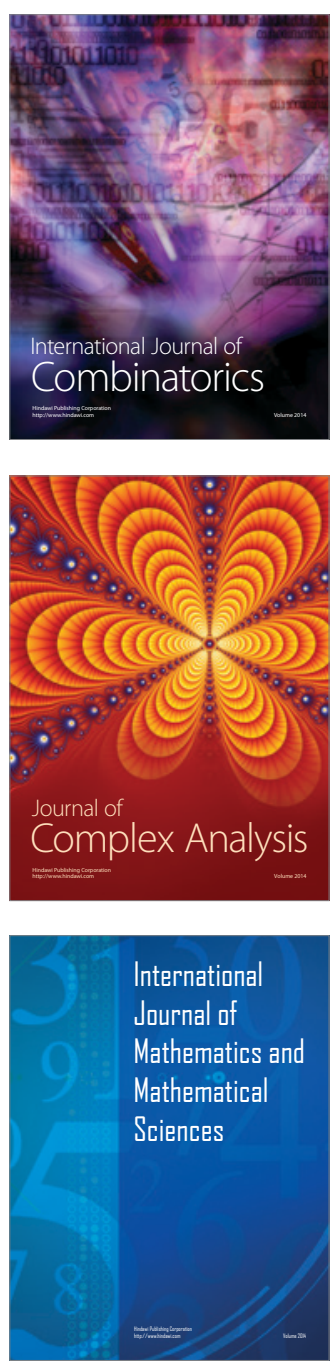
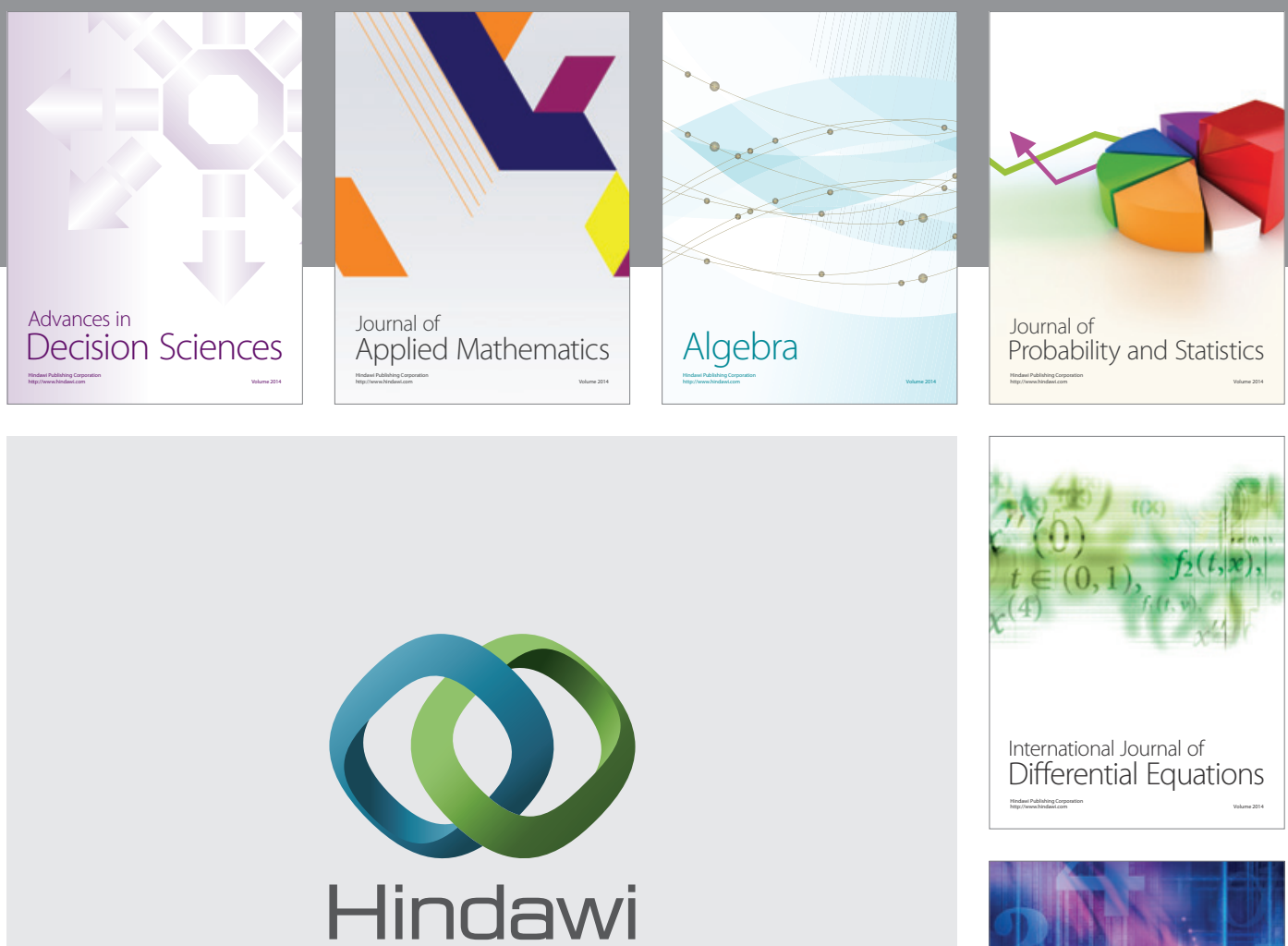

Submit your manuscripts at http://www.hindawi.com
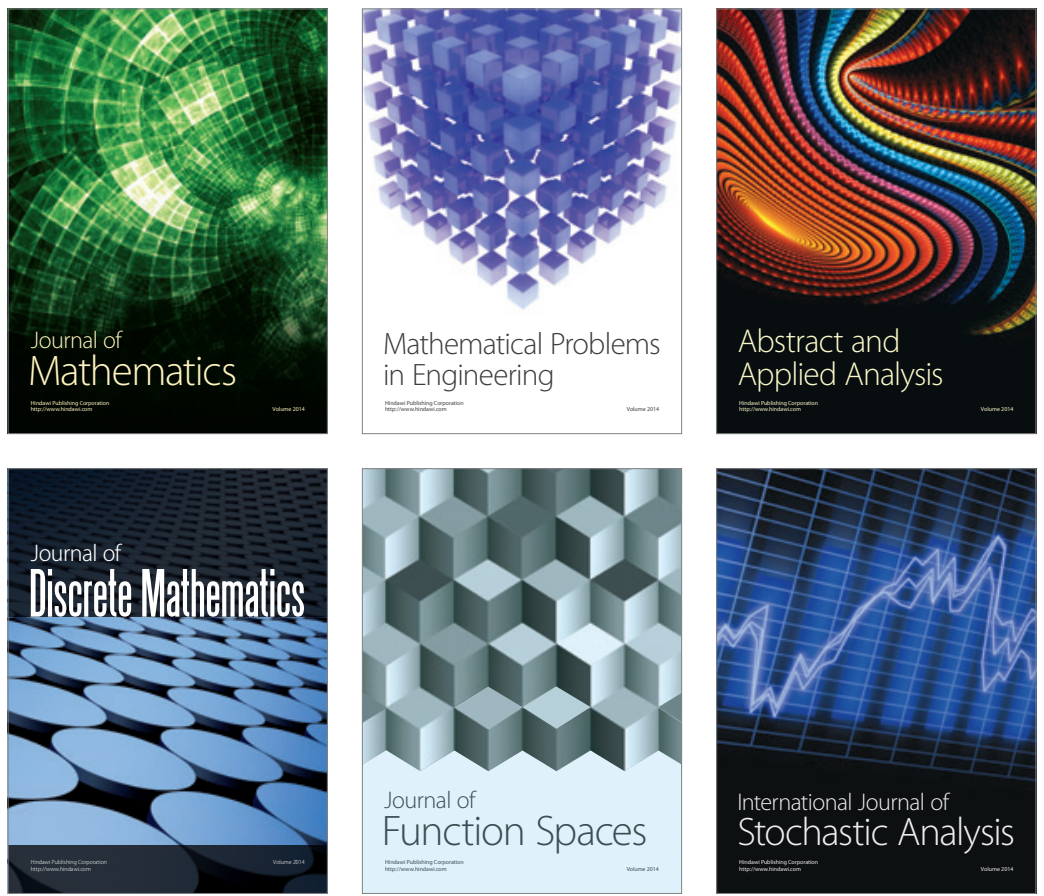

Journal of

Function Spaces

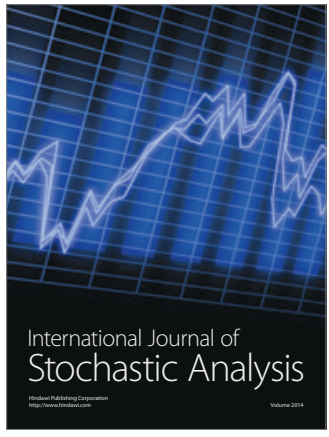

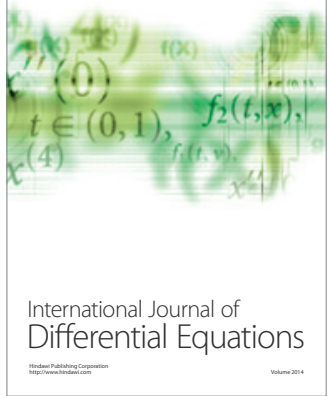
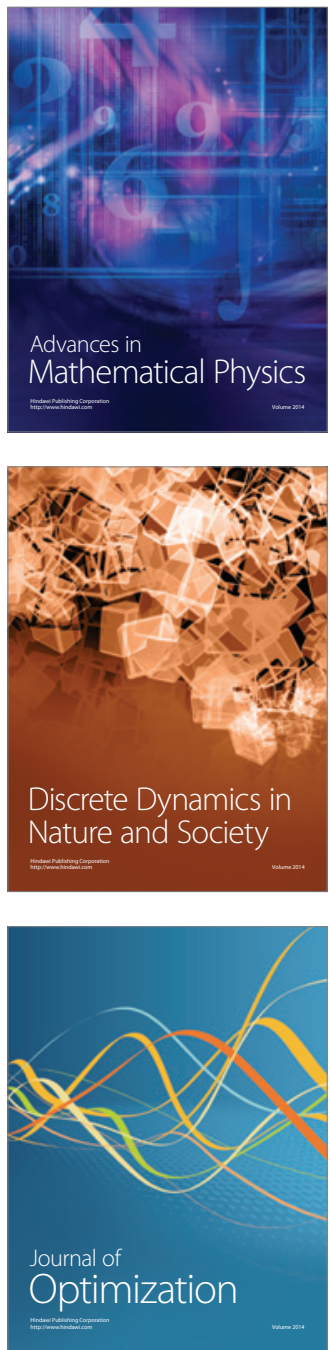